\title{
Emerging fractal patterns in a real 3D cerebral aneurysm
}

\author{
Gábor Závodszkyª,*, György Károlyi ${ }^{\mathrm{b}}$, György Paál ${ }^{\mathrm{a}}$ \\ ${ }^{a}$ Department of Hydrodynamics Systems, Faculty of Mechanical Engineering, Budapest University of \\ Technology and Economy, Múegyetem rakpart 3., 1111 Budapest, Hungary \\ ${ }^{b}$ Institute of Nuclear Techniques, Budapest University of Technology and Economy, Múegyetem rakpart 3. \\ 1111 Budapest, Hungary
}

\begin{abstract}
The behaviour of biological fluid flows is often investigated in medical practice to draw conclusions on the physiological or pathological conditions of the considered organs. One area where such investigations are proven to be useful is the flow-related formation and growth of different pathologic malformations of the cerebro vascular system. In this work, a detailed study is presented on the effect of a cerebral aneurysm on blood transport inside a human brain artery segment. This malformation causes strong flow instabilities that drives the flow system towards chaotic behaviour. The emerging fractal structure and some of its measurable properties have been explored using a method that makes the measurement of these properties feasible even in complicated large three dimensional data sets. We find that, from the investigated chaos parameters, the information dimension turns out to be the most reliable parameter to characterize chaotic advection in the vicinity of the aneurysm sac. We propose that properties of chaotic mixing close to aneurysms might be relevant for the condition of this pathologic malformation.
\end{abstract}

Keywords: chaotic advection, CFD, information dimension

\section{Introduction}

Numerous biological and chemical processes take place in fluid flows, ranging from environmental processes (plankton blooming $[1,2,3]$ or ozone hole formation $[4,5]$ ), through chemistry $[6,7,8]$, cavitation processes [9], valve flows [10], and combustion [11, 12], to microfluidics $[13,14]$. It has been shown [15] that mixing in fluids has an important influence on the biological or chemical activity that takes place in the fluid. The simplest approach to shed light on the properties of mixing in a fluid flow is to consider a passive scalar field, that is, particles of negligible size and mass that take on the velocity of the fluid instantaneously without inertia. The equation of motion of such particles is therefore $\dot{\mathbf{r}}(t)=\mathbf{v}(\mathbf{r}, t)$, where

\footnotetext{
${ }^{*}$ Corresponding author.

Email addresses: zavodszky@hds.bme.hu (Gábor Závodszky), karolyi@reak.bme.hu (György

Károlyi), paal@hds.bme.hu (György Paál)

URL: http://www.zavodszky.com (Gábor Závodszky)
} 
$\mathbf{r}(t)$ is the position of the particle at time $t$ and $\mathbf{v}(\mathbf{r}, t)$ is the velocity field of the fluid. Even for laminar time periodic flows, the solution to this equation is typically chaotic $[16,17,18]$, the particle trajectories deviating from the streamlines causing strong but non-turbulent mixing. This kind of complex behaviour in fluid flows is called chaotic advection [17].

Open flows constitute a large class of fluid flows where fluid is continuously transported into a certain region of observation and out of it. In these flows, a part of the advected particles can get trapped for a long time within the region of observation. The majority of the particles leave the region of observation in a short time, but those that remain for a long time accumulate along a filamentary fractal pattern $[15,19]$ representing the unstable manifold of a chaotic set, the latter consisting of unstable (saddle type) periodic and non-periodic particle trajectories enclosed in the region of observation. The particle paths forming the chaotic set, despite being unstable, are accessible from special initial conditions that form its stable manifold. Trajectories having started from the stable manifold approach the chaotic set and get trapped in the region of observation.

The fractal dimension $D_{0}$ of the unstable manifold gives information on the geometry of the patterns traced out by the advected particles while the information dimension $D_{1}$ also characterises the probability distribution (or relative density) of particles along the fractal unstable manifold [20]. The appearance of filamentary fractal patterns is the result of the stretching and folding action of the mixing fluid generated by the strong sensitivity on the initial conditions, the main characteristics of chaos. This strong sensitivity on initial conditions is characterised by the average Lyapunov exponent $\lambda$ describing the typical exponential rate of separation of initially close particles: $d(t)=d(0) e^{\lambda t}$ where $d(t)$ is the distance between the initially close particles at time $t[20]$. In open flows, another important number is the escape rate $\kappa$ which gives the rate of exponential decay of the number of particles still in the region of observation at time $t: n(t)=n(0) e^{-\kappa t}$. These quantities are not independent of each other, the relation $D_{0}=D-\kappa / \lambda$ holds between them where $D$ is the dimension of space in which the quantities are measured [20].

Chaotic advection can have important consequences in case of blood flow [21, 22, 23]. For instance, platelets are transported in the blood while they are biochemically active. It has been shown $[15,24,25]$ that the fractality of the pattern traced out by active particles dramatically modifies the reaction equations describing their activity. Productivity, which is proportional to the concentration of the chemical constituents in a well-mixed medium, turns out to depend on the information dimension of the distribution of the constituents in an open flow $[15,25]$. This dependence is of singular type, productivity is proportional to $c^{-\alpha}$ where $c$ is the average concentration of a species and $\alpha=\left(D_{1}-1\right) /\left(2-D_{1}\right)$ [24]. In the referred work, a two-dimensional idealisation was assumed, hence $1<D_{1}<2$ and $\alpha>0$. This implies that, in contrast to a well-mixed medium, the production is proportional to (nearly) the reciprocal of the concentration rather than the concentration itself. The reason behind the resulting advantage of rarity principle is that the fewer particles trace out the finer and finer details of the filamentary fractal structure of the unstable manifold, the longer the surface becomes between the chemical constituents. (Of course this principle is valid only as long as fractal patterns are traced out by the constituent.) An example is platelet activation which has been speculated [21] to follow similar rules. Hence, it is of great 
interest to obtain information on the fractal and information dimension of biochemically active particle patterns in blood flow.

In the current work, we concentrate on characterising the flow inside a real cerebral aneurysm geometry using the properties of chaotic advection. The studied geometry originates from the medical recording (CT angiography) of a frequent human arterial disease (3-5 \% in the total population $[26,27])$, a cerebral aneurysm. The flow in this geometry was simulated by an in-house high resolution CFD code based on the lattice Boltzmann method (LBM) that has been well tested and validated [28]. This simulated data set (velocity and pressure field) was the basis for further investigations. Cerebral aneurysms are pathogenic lesions on brain vessel walls. They typically appear behind highly curved sections or along the bifurcation points of the main cerebral blood supply, the Circle of Willis, which is responsible for carrying about $80 \%$ of the total blood volume circulating in the brain. From a clinical point of view, these aneurysms impose a high risk as their potential rupture could lead to brain hemorrhage, one of the main causes of stroke. Their medical treatment can carry a significant risk and they often impose a high strain on the human body. Therefore, it would be crucial for medical practitioners to be able to classify the aneurysms by the predictable risk of rupture. Based on this classification, the necessary approach for the treatment could then be decided. The most common classification was based on the mere size of the aneurysm [26], or some size ratio like the diameter of the aneurysmal neck compared to the largest diameter of the bulb. Later, strong indications have been found $[29,30,31,32]$ that the properties of the local blood flow has a significant impact on the aneurysmal behaviour. Thus a newer, more refined characterisation method was to correlate the rupture risk (among other features of the pathogenesis) to the local wall shear stress $[33,34,35]$ or the wall shear stress combined with the wall shear stress gradient [36]. In this way, some of the properties of the emerging blood flow are incorporated in the decision, not just the bare geometry. Further investigations suggested the importance of one particular property of the wall shear stress, its periodic change in time quantified by the so called oscillating shear index [37]. The most recent works bring a new concept called energy loss $[38,39]$ being defined as the difference in total kinetic energy of the flow inside the concerned vessel section between the aneurysmal and the reconstructed pre-aneurysmal state. These are just the main contenders for the improvement of the risk assessment, for a broader overview see ref.[40].

Though each of these classification methods shows some level of correlation with the ruptured or unruptured state of the examined aneurysms, the results are still controversial [39] and it seems that none of them shows high enough statistical validity to give a basis to a robust and unequivocal decision making process. This can encourage further search for a more robust property of the vessel section that is quantifiable and is in a strong connection with both the geometry and the emerging flow field. Following the lead of the presented information, in this work the presence of chaotic structures in a pathologic vessel section is investigated through the examination of the trajectories of massless tracer particles. One of our main results is that the particle transport within and around real 3D cerebral aneurysms exhibits chaotic properties and can be described using fractal characteristics. We propose that the information dimension $D_{1}$ is a reliable chaos parameter that is both simple to 
compute and robust to characterise properties of chaotic advection in cerebral aneurysms.

\section{Methods}

\subsection{Flow field}

Real blood vessels usually have highly curved and irregular shapes. The layout of the vessel section used during the current work was obtained from CT (X-ray computed tomography) angiography [41]. The flow field of the blood was obtained from an experimentally validated CFD (computational fluid dynamics) simulation [28] that used the lattice Boltzmann method [42] with a very fine numerical grid in order to handle the aforementioned highly curved and irregular nature of the geometry. Figure 1. shows the layout of the vessel geometry and gives an impression of the sensitive dependence of the particle trajectories and initial conditions by showing the trajectories of three tracers initiated very close to each other (with under $10^{-4} \mathrm{~mm}$ initial distance between them). The obtained time-varying flow field represents the realistic blood flow during a full cardiac cycle. During the CFD computation, the vessel wall was considered to be a rigid, no-slip surface. On the inlet and on the two larger outlets, time-dependent volumetric flow rates were prescribed that matched a realistic curve during a full cardiac period. This function is the same as the one used in Refs. [28] and [41]. The third, smaller outlet was defined as a constant pressure boundary to avoid over-constraining the model. The blood was approximated as a Newtonian fluid. The simulation domain (fluid and wall nodes combined) contained approximately $10^{7}$ numerical cells. The physical time-span of a simulated cardiac cycle was exactly $1 \mathrm{~s}$. During this time, 36 snapshots of the flow field were recorded at equal time-steps to be used later for the tracer path integration. For a more detailed description of the simulation parameters and the boundary conditions see [28], where the validation of the flow field results against experimental data [41] are also described. Once the flow field $v(r, t)$ is generated, it can be used to compute the path of passively advected particles from $\dot{r}(t)=v(r, t)$ numerically, as discussed in Subsection 2.3. Three such particle paths, initiated close to each other, are shown in Fig. 1. We see that, despite their initially small distance from each other, the particle paths deviate quite rapidly from each other, thus there is strong dependence on the initial conditions, which is a unique sign of chaos.

\subsection{Free-energy function}

One possible technique to measure important chaos characteristics in a relatively simple way is to take a large number of particle starting positions along a line segment of length $L$ which intersects the stable manifold of the chaotic set. Then we measure the residence time for each particle initial condition, that is, the time it takes for the particles to pass through the investigated flow domain. Then we can count the number $N(t)$ of intervals along $L$ with residence time longer than $t$.

Let $\ell_{i}(t)$ denote the length of these intervals along $L$ with $i=1,2, \ldots, N(t)$. This notation is explained with the help of Fig. 2a and Fig. 2b. For a large enough $t$ the free 


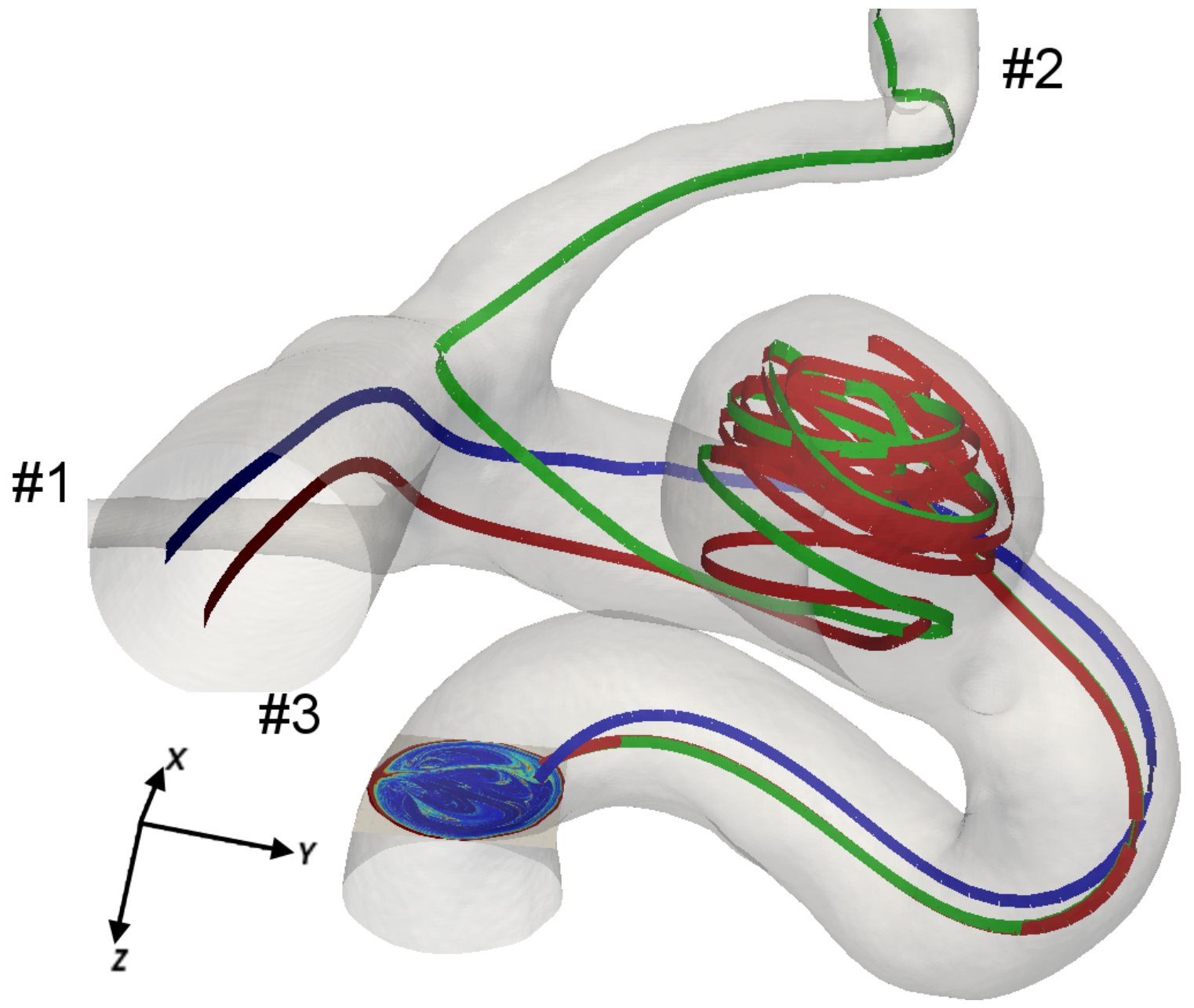

Figure 1: The layout of the aneurysm and the investigated vessel segment. The surface shows the surface of the vessel lumen. The inlet can be found at the bottom of the figure while the other openings are outlets numbered in the decreasing order of their diameter. The coloured paths are the computed trajectories of three particles started from the cross-section at the inlet from close proximity (within $10^{-4} \mathrm{~mm}$ ). Their quick separation is an indication to the high sensitivity to initial conditions which is a usual sign of chaotic behaviour. 
a.)
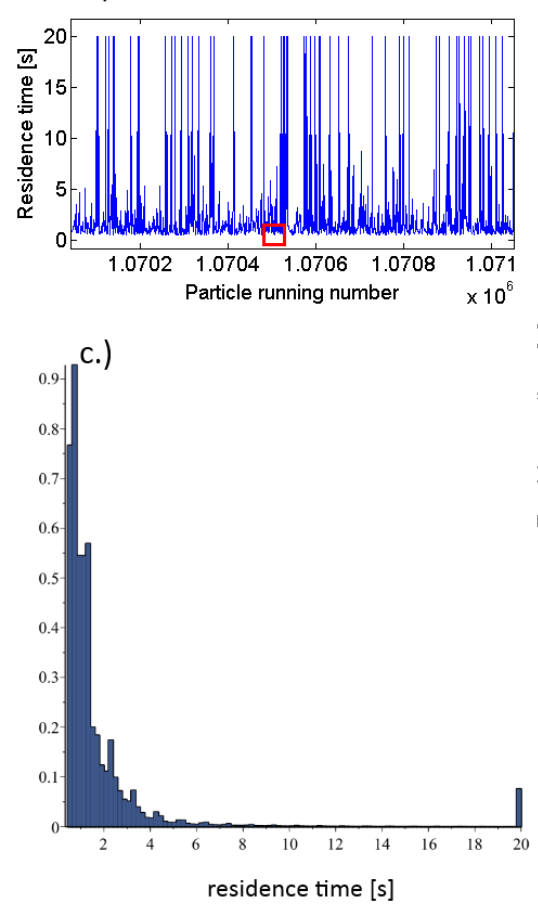

b.)

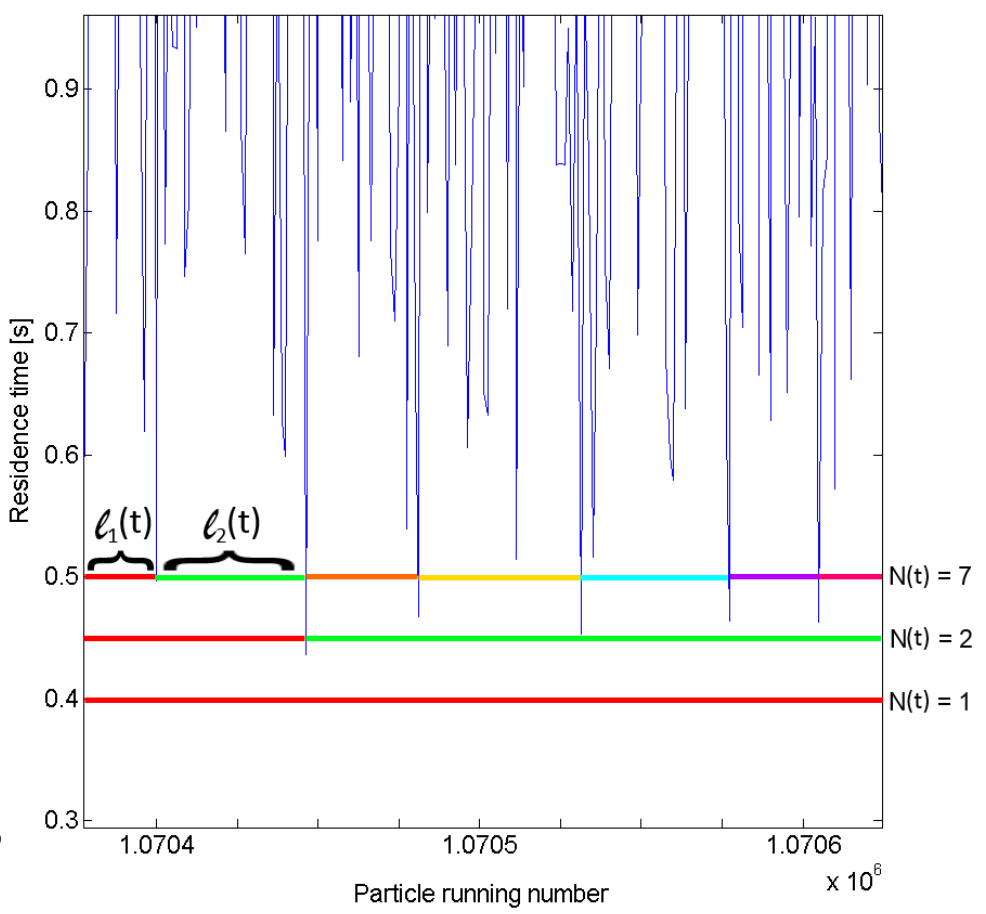

Figure 2: (a) Residence time as a function of the initial position along a line section of length $L$. (b) The number $N(t)$ of intervals of length $\ell_{i}(t)$ at three different residence time levels within the enlarged area of the small red rectangle indicated in (a). (c) The distribution of residence times of particles initiated from the plane shown in Fig. 1. The computation is run for up to $20 \mathrm{~s}$, the peak at $20 \mathrm{~s}$ shows the number of particles still inside the domain. 
energy function $F(\beta)$ can be defined as in [43]

$$
e^{-\beta F(\beta) t} \propto \sum_{i=1}^{N(t)}\left(\ell_{i}(t)\right)^{\beta} .
$$

The important quantities describing chaotic advection can be derived from this function, for instance,

$$
\begin{gathered}
\kappa=\left.\beta F(\beta)\right|_{\beta=1}, \\
\lambda=\left.\frac{d \beta F(\beta)}{d \beta}\right|_{\beta=1}, \\
0=\left.\beta F(\beta)\right|_{\beta=D_{0}}, \\
D_{1}=\left.\frac{d F(\beta) / d \beta}{d[\beta F(\beta)] / d \beta}\right|_{\beta=1} .
\end{gathered}
$$

\subsection{Computation of chaotic properties}

The accuracy of the chaotic properties computed through the free-energy function formalism depends on the accuracy of the calculated particle trajectories and the accuracy of the numerical evaluation of the $\beta F(\beta)$ function, which in turn depends on the number of computed trajectories initiated along a given line segment. Thus, it seems natural to elaborate on the accuracy of these two computations. The time-dependent velocity field described previously is used to calculate the paths of passive tracer particles of negligible size and mass. Based on the mean flow velocity (approximately $0.3 \mathrm{~m} / \mathrm{s}$ ), the tracers starting from the inlet and following the centreline should leave the geometry through one of the outlets in about $1.1 \mathrm{~s}$. Most particles take more time to leave as there are slower than the average portions of the flow and the pathlines rarely follow the centerline. For this reason, the computations are usually run for up to 20 heart beat cycles (equal to $20 \mathrm{~s}$ ). The computation of the paths requires high numerical precision during the integration, as the flow field has several strongly unstable regions. These regions can make adjacent particles to diverge quickly, thus, a small inaccuracy in their position can produce rather different trajectories. Typical unstable regions are the highly curved portions and the branching points, but the most vigorous one is around the mouth of the aneurysmal sac. The effect of these unstable regions is observable in Figure 1 as these are the typical points where the three trajectories separate. As a result of the sensitivity to initial conditions, a very small timestep has to be used during the integration process. For the integration the 4th order Runge-Kutta method is chosen; due to the very small timesteps stability is not an issue for this explicit method. The derivative function (the local velocity of the tracer particles) was evaluated using linear interpolation among the eight nearest grid points enclosing the actual point in space and also between the two nearby snapshots in time. Then the residence time is measured as the time needed by the particles initiated from the inlet cross-section to reach an outlet.

We test the convergence by measuring how the information dimension $D_{1}$ depends on the integration time step (Fig. 3a) and on the number of trajectories initiated along a line 
segment to compute the free energy function $F(\beta)$ (Fig. 3b). To compute Fig. 3a, the density of the traced trajectories is set to a high value: $8 \times 10^{5}$ particles on a $3 \mathrm{~mm}$ line segment, which means the distance between the starting positions of the particles is $3.75 \times 10^{-9} \mathrm{~m}$. A high value in our context means the highest sensible achievable value using the computational resources available to us. For the evaluation of the $\beta F(\beta)$ function with different number of trajectories in Fig. 3b., the number of time divisions between two snapshots was set to 600 . We used the same test line segment gradually increasing the number of particles launched along the line segment in each run. Figure $3 \mathrm{~b}$ summarises the results of those runs.
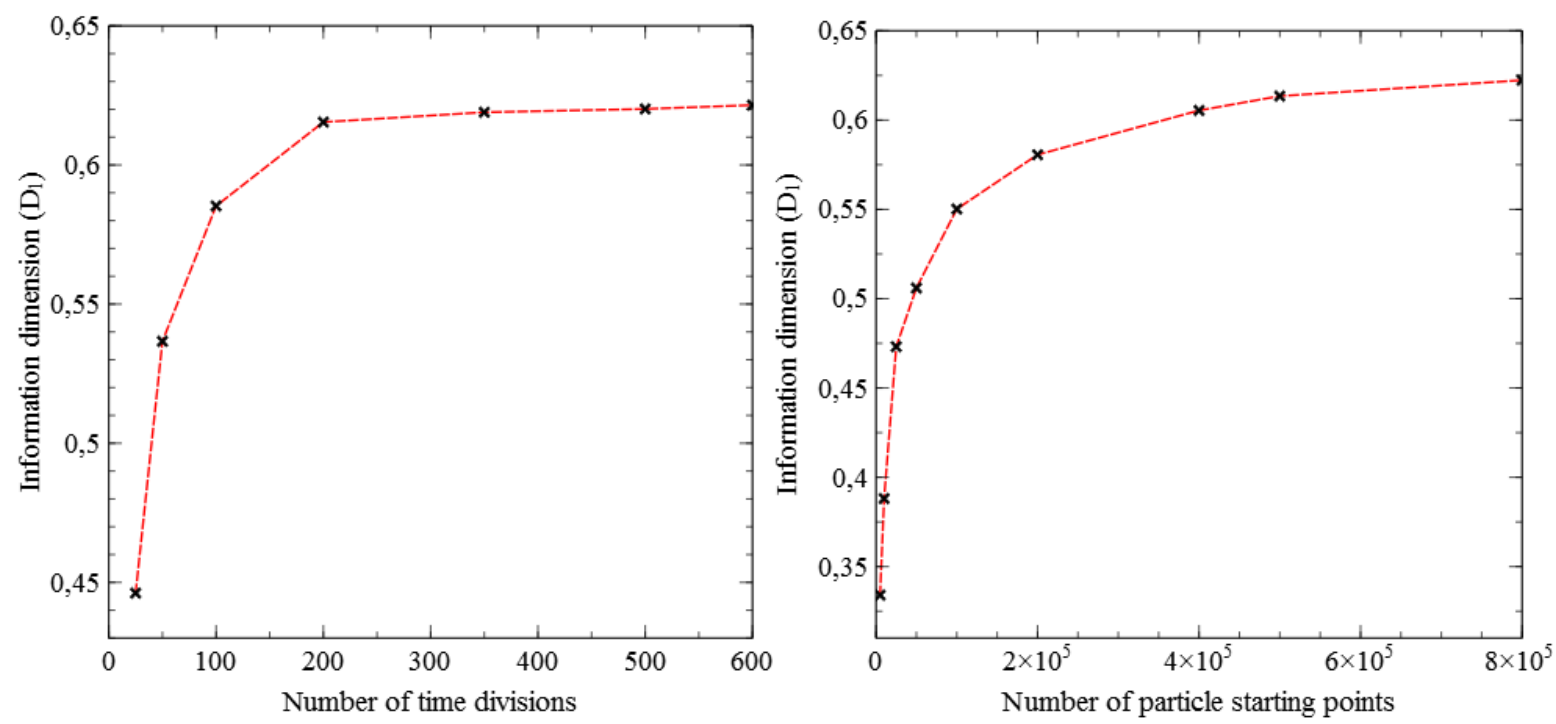

Figure 3: The information dimension along the test line segment denoted 'b' in Figure 4 as a function of (a) the number of the time divisions between two flow field snapshots, and (b) the number of trajectories initiated along the line segment.

The number of time divisions is set to 500 during the computations; that is, the time length of $\frac{1}{36} \mathrm{~s}$ is further divided into 500 equal intervals resulting in a time step of $5.5 \times 10^{-5} \mathrm{~s}$ for the integration. The finally applied particle starting point density is $5 \times 10^{5}$ particles placed equidistantly along the $3 \mathrm{~mm}$ long test segment, implying a distance of $6 \times 10^{-9} \mathrm{~m}$ between the starting positions of these particles.

\section{Results}

\subsection{Qualitative results}

First we compile a qualitative image of the sensitivity to initial position of trajectories. Starting from a planar cross-section of the inlet section (its position is shown in Fig. 1), four million randomly placed trajectory paths are computed. In Figure 4 the starting position of each particle on the inlet cross-section is coloured according to the outlet through which the particle leaves the domain. The image shows a wild variation of the final positions of even those particles that started close to each other. 


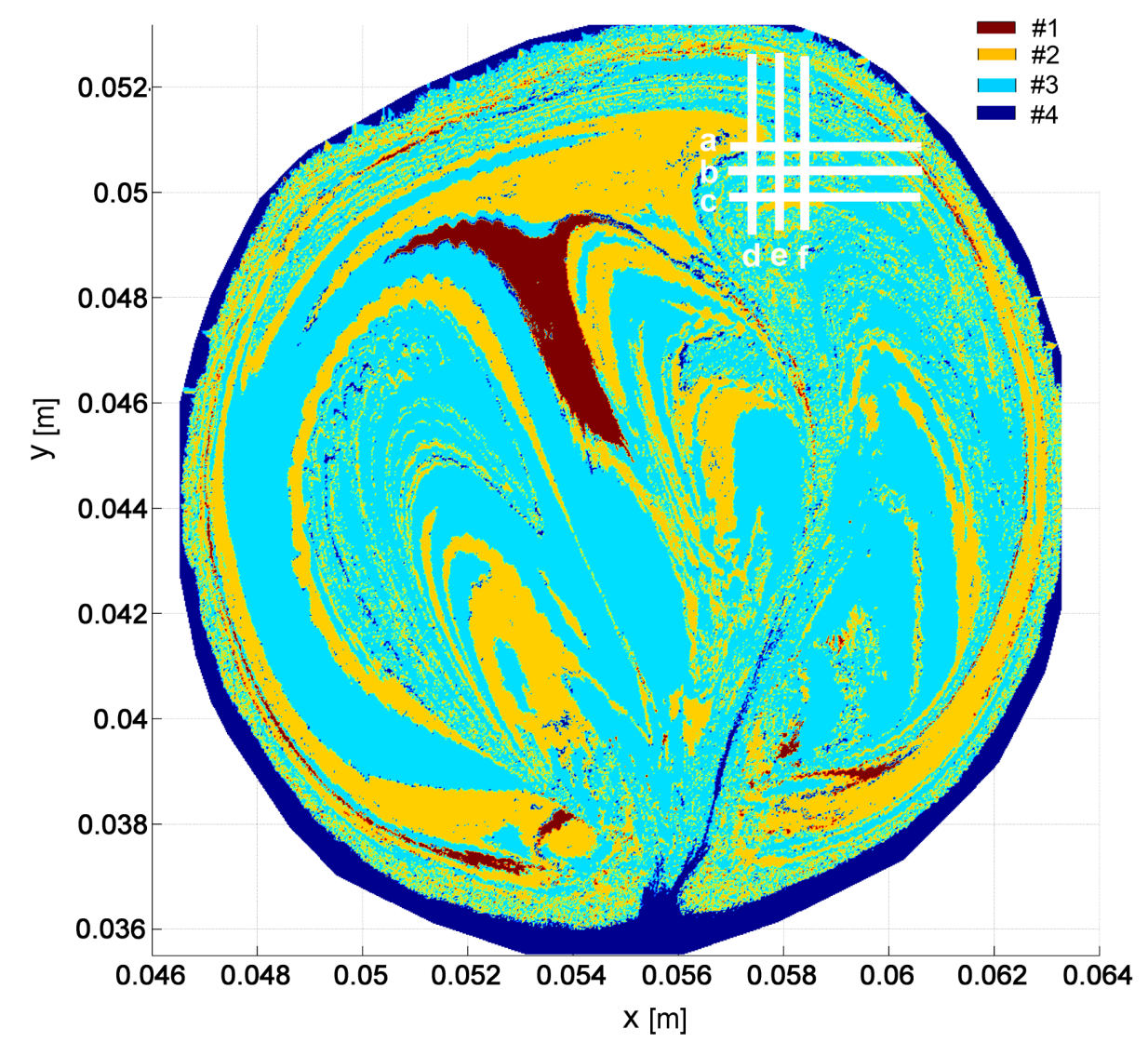

Figure 4: The cross-section of the inlet vessel from where the tracers were initiated. The colours \#1, \#2, and \#3 represent the different outlets through which the particles (started from the given position) leave the domain. The corresponding outlet positions are shown in Fig. 1 with the same numbering. Colour \#4 accounts for the particles still inside the domain after $20 \mathrm{~s}$. The white line segments marked "a" through "f" denote the locations for the computations of $D_{1}$, c.f. Table 1.

Strong dependence of the outlet through which particles leave on the initial position, as shown in Fig. 4, is expected to imply similar variations in the residence times. Figure 2c shows the distribution of the residence times for the same particles followed in Fig. 4 . We see that many particles leave the domain of the investigated vessel section quite rapidly. There are, however, particles that spend more than 20 s, i.e., 20 heartbeats in the domain. Note that the particles stuck at the vessel wall during their advection are filtered out from the shown distribution. Hence the particles that spend a very long time in the domain must be those that are trapped in the vicinity of the chaotic set. This set, though it consists of a zero measure set of unstable trapped orbits, is accessible from the direction of its stable manifold [20]. The particles initially close to the stable manifold of the chaotic set get close to the orbits of the chaotic set, follow them for long time before reaching any of the outlets. Physically, these are particles that "hesitate" for a long time which outlet to take eventually. The trajectories exactly on the stable manifold form the boundary between the basins of attraction of the outlets (Fig. 4.). Initial conditions exactly on the boundary, on the stable 
manifold of the chaotic set, never leave the investigated domain [20].

In order to obtain information on the spatial distribution of the initial positions leading to long residence times, we measure the residence time for each initial point used in Fig. 4. Then, using colour coding in Fig. 5, we plot the residence time on the initial position.

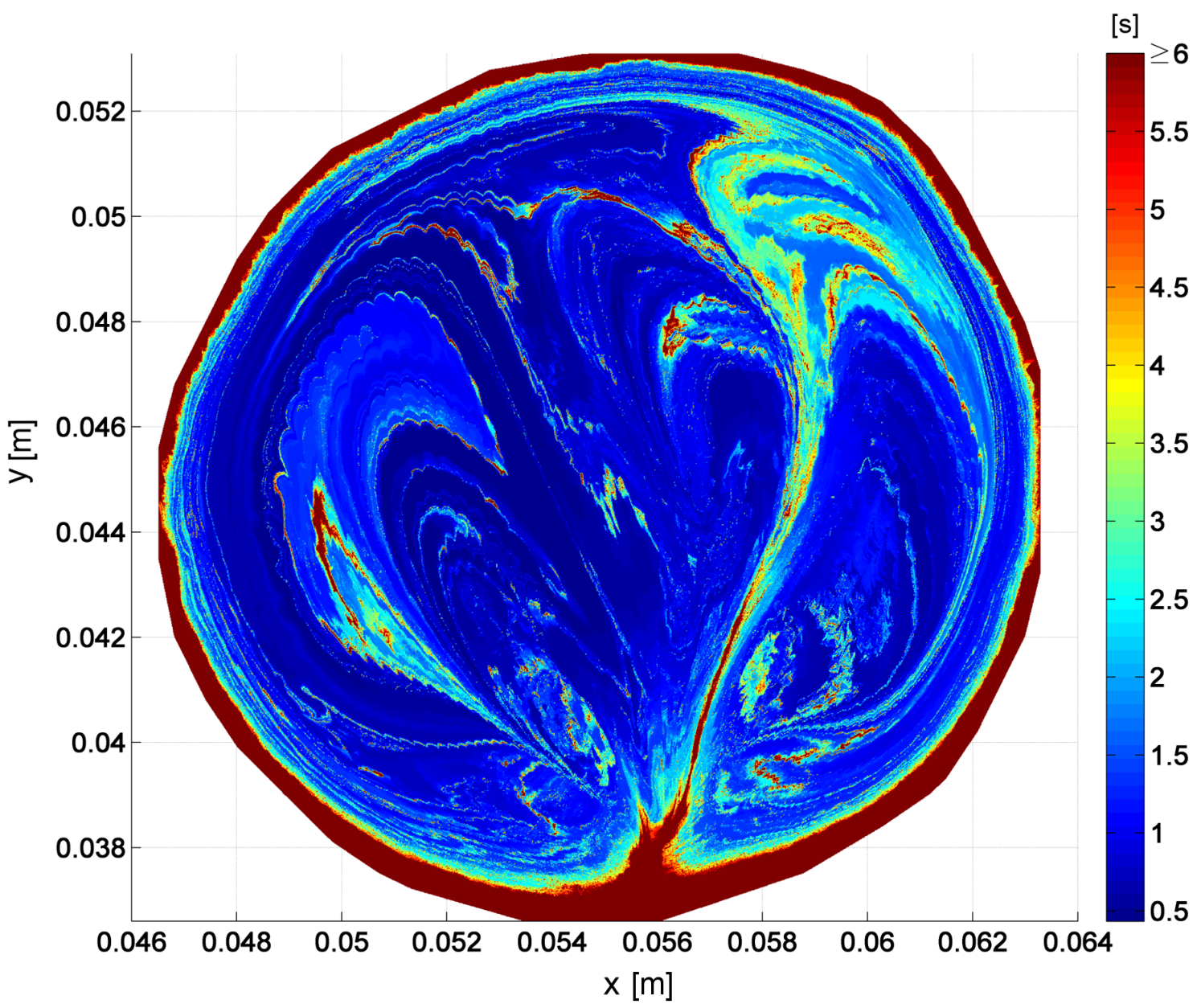

Figure 5: Using colour coding, the residence times of the particles are indicated at their starting positions on the inlet cross-section. Though the calculation of residence times are done up to 20 cardiac cycles (c.f. Fig. 2c), these residence time values on the colour scale are cut at the upper limit of $6 \mathrm{~s}$ (6 cardiac cycles) to provide a better visual representation.

A portion of the trajectories with high residence times start from right beside the vessel wall. This is not surprising, because blood velocity is low here due to the no-slip boundary condition on the vessel wall. Inside the vessel, however, we find starting points from where the tracer trajectories approach the vicinity of the chaotic set. At these points the residence times are much higher than $1.1 \mathrm{~s}$, the time the particles would need to pass the investigated vessel segment, were they traveling at the average blood speed along the centerline of the 
blood vessel. The initial positions for particles with long residence times trace out intricate, filamentary patterns in Fig. 5. Such patterns have been observed previously in other fluid flows $[19,44]$, where they turned out to be fractal patterns generated by the transient chaotic motion of the particles.

\subsection{Quantitative results}

We use the free-energy formalism to extract chaotic properties of mixing in the blood flow of the investigated vessel segment containing the aneurysm. We choose line segments in the plane of the inlet cross-section shown in Figure 4. The locations of these line segments are selected so that they lie in the $x \in[0.056,0.061], y \in[0.048,0.052]$ region. Indeed, this part of the cross-section seems to be the most rich in intricate patterns. Three of the selected line segments are parallel to the $x$ axis, while the other three are perpendicular to it. The extracted quantities are shown in Table 1.

\begin{tabular}{ccccc}
\hline & $D_{1}$ & Lyapunov $(\lambda)$ & Escape rate $(\kappa)$ & line \\
\hline \multirow{3}{*}{ parallel to $x$} & 0.6233 & 3.3841 & 1.3011 & $\mathrm{a}$ \\
& 0.6025 & 4.4728 & 1.2156 & $\mathrm{~b}$ \\
& 0.6427 & 3.3882 & 0.7889 & $\mathrm{c}$ \\
\hline \multirow{3}{*}{ parallel to $y$} & 0.6446 & 2.2799 & 0.9486 & $\mathrm{~d}$ \\
& 0.6098 & 2.0528 & 0.8935 & $\mathrm{e}$ \\
& 0.6143 & 2.6301 & 0.9918 & $\mathrm{f}$ \\
\hline
\end{tabular}

Table 1: Quantities measured from the $\beta F(\beta)$ function along six different line segments indicated in Fig. 4. The quantities $D_{1}, \lambda$ and $\kappa$ are computed from Eq. 5, 3, and 2, respectively.

Throughout the computation the information dimension $D_{1}$ of the intersection of the stable manifold with the chosen line segment proved to be the most stable quantity. Based on these six measurements, its average is $\overline{D_{1}}=0.623$ with a standard deviation of $\sigma=0.017$. The other quantities show much more spatial variability. Hence we propose that from all computed quantities the information dimension $D_{1}$ is the most robust parameter to describe mixing and chaotic advection in such diseased blood vessel segments.

There are several other points of instability in the flow apart from the aneurysm where the flow profile changes rapidly, like the bifurcation points or high curvature parts of the blood vessel. As particles can also get trapped there, we also measure the residence time of particles starting from the same initial plane, but only until they reach the distal neck of the aneurysm. This way, the effect of further flow instabilities downstream of the aneurysm can be excluded from the residence time and the fractal characteristics.

The resulting residence time plot is shown in Fig. 6, which has the same main features as Fig. 5. We also measure the information dimension average $\overline{D_{1}^{\prime}}$ in this plot, and it turns out to be within the standard deviation $\sigma$ from the previous average $\overline{D_{1}}\left(\overline{D_{1}^{\prime}}=0.607\right.$ and $\sigma^{\prime}=0.009$ ). Finally, yet another measurement downstream of the aneurysm neck is carried out but the residence time does not show such variation as that in Fig. 5, no fractal patterns emerged. This strongly suggests that the chaotic structure is mainly generated by 
the presence of the aneurysm, and it is robust enough that it suppresses the effect of other smaller instabilities along the inspected geometry.

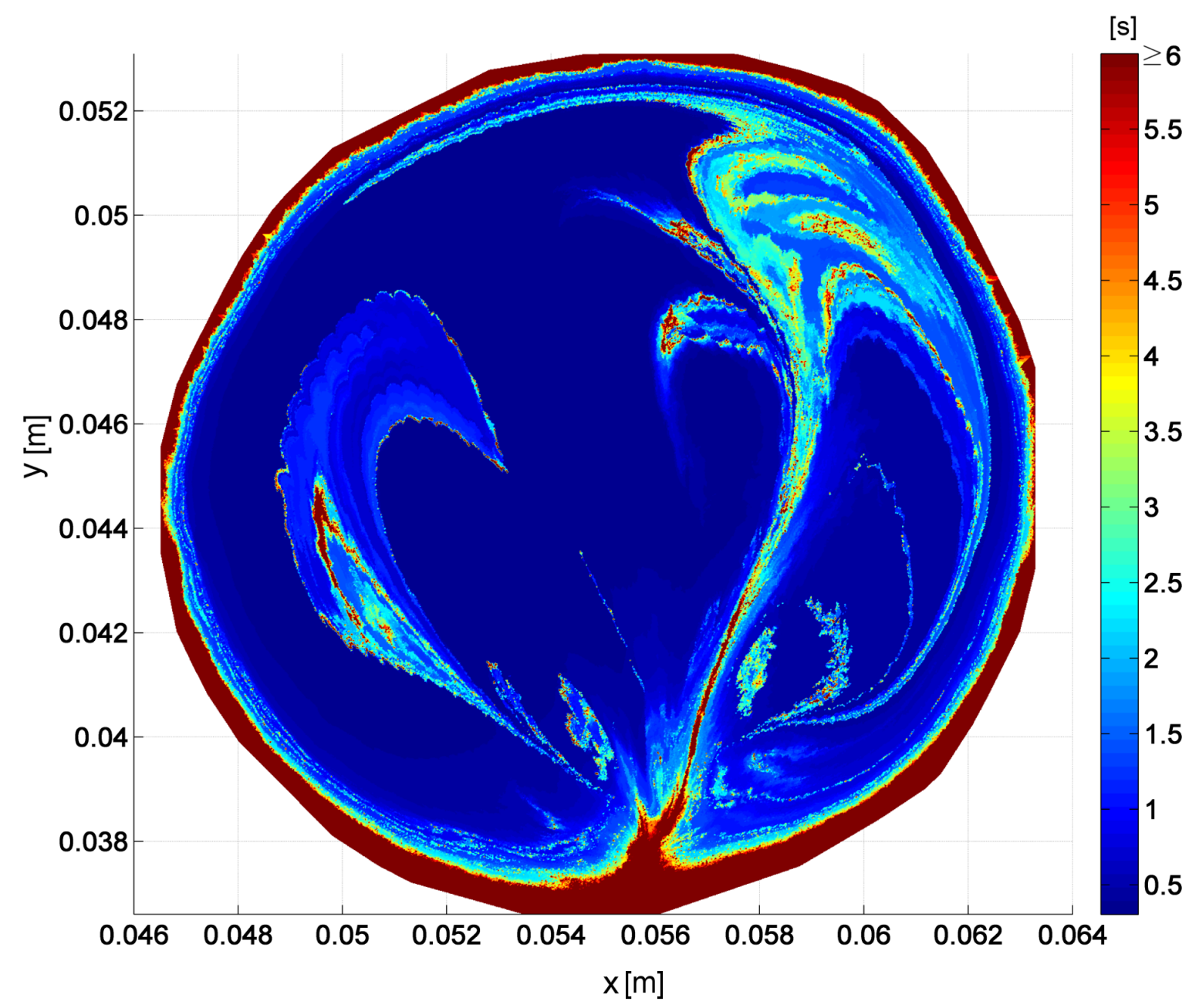

Figure 6: Residence time of the particles until they pass the neck of the aneurysm is indicated with colour coding on their initial position. The colour scale is the same as that in Fig. 5.

\section{Conclusions}

The aneurysm as a pathologic change in the vessel geometry causes a major disturbance in the flow field creating vortices and possibly other instabilities. These instabilities modify the paths of some of the particles residing in the flow resulting in an anomalous increase in their residence time. The modified paths bear the characteristics of chaotic dynamics. Several quantities related to chaos (most importantly the information dimension) can be measured using the methods shown in this work. Though further measurements on different geometries will be needed to justify it, but the results upon this geometry support the 
idea that the chaotic behaviour inflicted by the aneurysm sac is quite dominant. In the current case the flow disturbances other than the aneurysm sac itself are not relevant in the measurement of $D_{1}$. This might make regular clinical measurements feasible as the actual nearby geometry and flow disturbance seems not to modify the trapping effect of the

aneurysm. Several other interesting questions arise that require further investigation. It is possible that this chaotic behaviour or even some of its statistical description (like $D_{1}$ ) could be linked to the formation or rupture process of the aneurysms. For example, the increased residence times, efficient mixing and exponential outflow of the particles in the diseased blood vessel segment can have direct effects on the biochemical reactions taking place in blood $[21,22,23]$. It would also be meaningful to collect statistical information about the relation of pathological vessel malformations, and the fractal properties they enforce upon the flow. At the end, the fractal properties carry information on the aneurysm geometry in such a robust way that it seems useful to utilise them as an indicator during the medical decision-making and as a descriptor in the aneurysm classification process.

\section{Acknowledgements}

This work was financially supported by the New Szchenyi Development Plan (TMOP4.2.1/B-09/1/KMR-2010-0002). We also thank for the financial support by OTKA grants NK 100296 and K 100894".

[1] E. R. Abraham, "The generation of plankton patchiness by turbulent stirring," Nature, vol. 391, no. 6667 , pp. 577-580, 1998.

[2] A. B. Schelin, G. Krolyi, A. P. S. d. Moura, N. A. Booth, and C. Grebogi, "Fractal structures in stenoses and aneurysms in blood vessels.," Philosophical transactions. Series A, Mathematical, physical, and engineering sciences, vol. 368, no. 1933, pp. 5605-5617, 2010.

[3] A. Martin, "Phytoplankton patchiness: the role of lateral stirring and mixing," Progress in Oceanography, vol. 57, no. 2, pp. 125 - 174, 2003.

[4] S. Solomon, "Stratospheric ozone depletion: A review of concepts and history," Reviews of Geophysics, vol. 37 , no. 3, pp. 275-316, 1999.

[5] B. Eckhardt, "Integrable four vortex motion," Physics of fluids, vol. 31, no. 10, p. 2796, 1988.

[6] I. R. Epstein, "The consequences of imperfect mixing in autocatalytic chemical and biological systems," Nature, vol. 374, no. 6520 , pp. 321-327, 1995.

[7] M. Menzinger and P. Jankowski, "Heterogeneities and stirring effects in the belusov-zhabotinskii reaction," The Journal of Physical Chemistry, vol. 90, no. 7, pp. 1217-1219, 1986.

[8] G. Metcalfe and J. Ottino, "Autocatalytic processes in mixing flows," Physical review letters, vol. 72, pp. 2875-2878, 1994.

[9] F. Hegedüs, "Stable bubble oscillations beyond blakes critical threshold," Ultrasonics, vol. 54, no. 4, pp. 1113-1121, 2014.

[10] C. Hös and A. R. Champneys, "Grazing bifurcations and chatter in a pressure relief valve model," Physica D: Nonlinear Phenomena, vol. 241, no. 22, pp. 2068-2076, 2012.

[11] I. Kiss, J. Merkin, and Z. Neufeld, "Combustion initiation and extinction in a 2d chaotic flow," Physica D: Nonlinear Phenomena, vol. 183, no. 3, pp. 175-189, 2003.

[12] F. William, "Combustion theory: The fundamental theory of chemically reacting flow system," 1985.

[13] A. D. Stroock, S. K. Dertinger, A. Ajdari, I. Mezić, H. A. Stone, and G. M. Whitesides, "Chaotic mixer for microchannels," Science, vol. 295, no. 5555, pp. 647-651, 2002.

[14] S. D. Mller, I. Mezi, J. H. Walther, and P. Koumoutsakos, "Transverse momentum micromixer optimization with evolution strategies," Computers \& Fluids, vol. 33, no. 4, pp. 521 - 531, 2004. 
[15] T. Tél, A. de Moura, C. Grebogi, and G. Károlyi, "Chemical and biological activity in open flows: a dynamical system approach," Physics reports, vol. 413, no. 2, pp. 91-196, 2005.

[16] J. Ottino, "Mixing, chaotic advection, and turbulence," Annual Review of Fluid Mechanics, vol. 22, no. 1, pp. 207-254, 1990.

[17] H. Aref, "Stirring by chaotic advection," Journal of fluid mechanics, vol. 143, pp. 1-21, 1984.

[18] M. Zsugyel, T. Tél, and J. Józsa, "Numerical investigation of chaotic advection past a groyne based on laboratory flow experiment," Advances in Water Resources, vol. Accepted, no. 0, pp. -, 2014. Volume pending.

[19] G. Károlyi and T. Tél, "Chaotic tracer scattering and fractal basin boundaries in a blinking vortex-sink system," Physics Reports, vol. 290, no. 1, pp. 125-147, 1997.

[20] T. Tél and M. Gruiz, Chaotic dynamics: an introduction based on classical mechanics. Cambridge University Press, 2006.

[21] A. Schelin, G. Károlyi, A. De Moura, N. Booth, and C. Grebogi, "Chaotic advection in blood flow," Physical Review E, vol. 80, no. 1, p. 016213, 2009.

[22] A. B. Schelin, G. Károlyi, A. P. de Moura, N. A. Booth, and C. Grebogi, "Fractal structures in stenoses and aneurysms in blood vessels," Philosophical Transactions of the Royal Society A: Mathematical, Physical and Engineering Sciences, vol. 368, no. 1933, pp. 5605-5617, 2010.

[23] A. B. Schelin, G. Károlyi, A. P. de Moura, N. Booth, and C. Grebogi, "Are the fractal skeletons the explanation for the narrowing of arteries due to cell trapping in a disturbed blood flow?, Computers in biology and medicine, vol. 42, no. 3, pp. 276-281, 2012.

[24] Z. Toroczkai, G. Károlyi, Á. Péntek, T. Tél, and C. Grebogi, "Advection of active particles in open chaotic flows," Physical review letters, vol. 80, no. 3, p. 500, 1998.

[25] G. Károlyi and T. Tél, "Chemical transients in closed chaotic flows: the role of effective dimensions," Physical review letters, vol. 95, no. 26, p. 264501, 2005.

[26] G. J. Rinkel, M. Djibuti, A. Algra, and J. Van Gijn, "Prevalence and risk of rupture of intracranial aneurysms a systematic review," Stroke, vol. 29, no. 1, pp. 251-256, 1998.

[27] M. H. Vlak, A. Algra, R. Brandenburg, and G. J. Rinkel, "Prevalence of unruptured intracranial aneurysms, with emphasis on sex, age, comorbidity, country, and time period: a systematic review and meta-analysis," The Lancet Neurology, vol. 10, no. 7, pp. 626-636, 2011.

[28] G. Závodszky and G. Paál, "Validation of a lattice Boltzmann method implementation for a 3D transient fluid flow in an intracranial aneurysm geometry," International Journal of Heat and Fluid Flow, vol. 44, no. 0 , pp. $276-283,2013$.

[29] D. a. Steinman, J. S. Milner, C. J. Norley, S. P. Lownie, and D. W. Holdsworth, "Image-based computational simulation of flow dynamics in a giant intracranial aneurysm.," AJNR. American journal of neuroradiology, vol. 24, pp. 559-66, Apr. 2003.

[30] J. R. Cebral, M. a. Castro, J. E. Burgess, R. S. Pergolizzi, M. J. Sheridan, and C. M. Putman, "Characterization of cerebral aneurysms for assessing risk of rupture by using patient-specific computational hemodynamics models.," AJNR. American journal of neuroradiology, vol. 26, no. 10, pp. 2550-9, 2005.

[31] J. C. Lasheras, "The Biomechanics of Arterial Aneurysms," Annual Review of Fluid Mechanics, vol. 39, pp. 293-319, Jan. 2007.

[32] M. Piccinelli, A. Veneziani, D. a. Steinman, A. Remuzzi, and L. Antiga, "A framework for geometric analysis of vascular structures: application to cerebral aneurysms.," IEEE transactions on medical imaging, vol. 28, pp. 1141-55, Aug. 2009.

[33] M. Shojima, M. Oshima, K. Takagi, R. Torii, M. Hayakawa, K. Katada, A. Morita, and T. Kirino, "Magnitude and role of wall shear stress on cerebral aneurysm computational fluid dynamic study of 20 middle cerebral artery aneurysms," Stroke, vol. 35, no. 11, pp. 2500-2505, 2004.

[34] L. Boussel, V. Rayz, C. McCulloch, A. Martin, G. Acevedo-Bolton, M. Lawton, R. Higashida, W. S. Smith, W. L. Young, and D. Saloner, "Aneurysm growth occurs at region of low wall shear stress patient-specific correlation of hemodynamics and growth in a longitudinal study," Stroke, vol. 39, no. 11, pp. 2997-3002, 2008.

[35] D. M. Sforza, C. M. Putman, and J. R. Cebral, "Hemodynamics of cerebral aneurysms," Annual review 
of fluid mechanics, vol. 41, p. 91, 2009.

[36] Z. Kulcsár, A. Ugron, M. Marosfői, Z. Berentei, G. Paal, and I. Szikora, "Hemodynamics of cerebral aneurysm initiation: the role of wall shear stress and spatial wall shear stress gradient," American Journal of Neuroradiology, vol. 32, no. 3, pp. 587-594, 2011.

[37] T. Kawaguchi, S. Nishimura, M. Kanamori, H. Takazawa, S. Omodaka, K. Sato, N. Maeda, Y. Yokoyama, H. Midorikawa, T. Sasaki, et al., "Distinctive flow pattern of wall shear stress and oscillatory shear index: similarity and dissimilarity in ruptured and unruptured cerebral aneurysm blebs: Clinical article," Journal of neurosurgery, vol. 117, no. 4, pp. 774-780, 2012.

[38] Y. Qian, H. Takao, M. Umezu, and Y. Murayama, "Risk analysis of unruptured aneurysms using computational fluid dynamics technology: preliminary results," American Journal of Neuroradiology, vol. 32, no. 10, pp. 1948-1955, 2011.

[39] H. Takao, Y. Murayama, S. Otsuka, Y. Qian, A. Mohamed, S. Masuda, M. Yamamoto, and T. Abe, "Hemodynamic differences between unruptured and ruptured intracranial aneurysms during observation," Stroke, vol. 43, no. 5, pp. 1436-1439, 2012.

[40] M. Zanaty, N. Chalouhi, S. I. Tjoumakaris, L. Fernando Gonzalez, R. H. Rosenwasser, and P. M. Jabbour, "Aneurysm geometry in predicting the risk of rupture. a review of the literature," Neurological research, vol. 36, no. 4, pp. 308-313, 2014.

[41] Á. Ugron, M.-I. Farinas, L. Kiss, and G. Paál, "Unsteady velocity measurements in a realistic intracranial aneurysm model," Experiments in fluids, vol. 52, no. 1, pp. 37-52, 2012.

[42] S. Succi, The Lattice Boltzmann Equation for Fluid Dynamics and Beyond. Numerical Mathematics and Scientific Computation, Clarendon Press, 2001.

[43] T. Tél, "Fractals, multifractals, and thermodynamics," Z. Naturforsch, 43a, vol. 1154, 1988.

[44] C. Jung, T. Tél, and E. Ziemniak, "Application of scattering chaos to particle transport in a hydrodynamical flow," Chaos: An Interdisciplinary Journal of Nonlinear Science, vol. 3, no. 4, pp. 555-568, 1993. 